\title{
Cooccurrence violence conjugale, santé mentale ou consommation : mieux répondre aux besoins des familles
}

\author{
Geneviève Lessard \\ Université Laval \\ Chantal Lavergne \\ CIUSSS du Centre-Sud-de-l'Île-de-Montréal \\ Sylvie Lévesque \\ Université du Québec à Montréal \\ Annie Dumont, et Pamela Alvarez-Lizotte \\ Université Laval \\ Valérie Meunier \\ G.A.P.I. \\ Sophie M. Bisson \\ Université Laval
}

Geneviève Lessard, École de travail social et de criminologie, Université Laval, Québec, Québec; Chantale Lavergne, Centre intégré universitaire de la santé et des services sociaux du Centre-Sud-de-l'Ile de Montréal, Montréal, Québec; Sylvie Lévesque, Département de sexologie, Université du Québec à Montréal, Montréal, Québec; Annie Dumont, doctorante en travail social à l'Université Laval, Québec, Québec; Pamela Alvarez-Lizotte, doctorante en travail social à l’Université Laval, Québec, Québec; Valérie Meunier, Groupe d'aide aux personnes impulsives, Québec, Québec; Sophie M. Bisson, professionnelle de recherche à l’Université Laval, Québec, Québec.

Cette recherche a été rendue possible grâce à une subvention du Fonds de recherche du Québec - Société et culture et du ministère de la Santé et des services sociaux, dans le cadre du Programme d'actions concertées sur la violence conjugale. Les auteures souhaitent également remercier Mylène Bigaouette, Louise Riendeau, Mélanie St-Laurent et Marjolaine Lord pour leur appui au bon déroulement des différentes étapes du volet qualitatif du projet.

Pour toute correspondance concernant cet article, s'adresser à Geneviève Lessard, École de travail social et de criminologie, Pavillon Charles-De-Koninck, 1030, avenues des Sciences-Humaines, bureau 6417, Québec (Québec).

Courriel : genevieve.lessard@tsc.ulaval.ca 


\title{
RÉSUMÉ
}

Cette recherche qualitative explore les points de vue de mères, de pères et d'adolescents $(n=43)$ quant aux services reçus pour les problèmes cooccurrents de violence conjugale, de santé mentale et de consommation chez les parents. Les données ont été recueillies par des entrevues semi-dirigées, soutenues d'un outil de cartographie des services utilisés. L'analyse de contenu thématique montre que les participants déploient plusieurs stratégies pour faire face aux problèmes cooccurrents vécus et utilisent diverses ressources d'aide formelle et informelle. Plusieurs recommandations sont proposées pour améliorer l'offre d'aide aux familles en situation de cooccurrence, dont la concertation entre les différentes ressources concernées.

Mots clés : violence conjugale, santé mentale, consommation, cooccurrence, parentalité

\begin{abstract}
This qualitative research explores the point of views of mothers, fathers, and teenagers $(n=43)$ about the services they received for co-occurring problems of intimate partner violence, mental health, and parental substance use. The data were collected through semi-structured interviews, supported by a mapping tool of the services they used. The thematic content analysis shows that participants deploy several strategies to address co-occurring problems and use various formal and informal support resources. Several recommendations are proposed to improve the offer of assistance to families in situations of co-occurrence, including concertation between the different concerned resources.
\end{abstract}

Keywords: intimate partner violence, mental health, substance use, co-occurrence, parenthood

Les recherches montrent des taux de cooccurrence élevés entre la violence conjugale (VC) et les problèmes de santé mentale (PSM) ou de consommation (PC) d'alcool ou de drogues (Bromfield, Lamont, Parker \& Horsfall, 2010 ; Choenni, Hammink \& van de Mheen, 2017 ; Cleaver, Unell \& Aldgate, 2011). Une étude récente réalisée auprès de 61 mères et de 56 pères en protection de la jeunesse (Estefan et al., 2013) montre que $30 \%$ des mères et $26 \%$ des pères vivent à la fois un problème de $\mathrm{VC}$ et de toxicomanie et que $29 \%$ des mères et $23 \%$ des pères vivent à la fois un problème de VC et de santé mentale. La cooccurrence des 3 problèmes n'est pas documentée de façon spécifique. Bien qu'ils soient fréquents, les liens entre ces différents problèmes sont complexes et multidirectionnels. Par exemple, la VC est associée, chez les victimes, à de la dépression, du stress posttraumatique et des PC. De même, ces PC et PSM peuvent agir comme facteurs de risque et contribuer à aggraver les épisodes de violence et leurs conséquences (Galvani, 2004; OMS, 2006). De plus, la cooccurrence de ces problèmes accroît la probabilité que les enfants vivant dans ces familles soient maltraités (Bromfield et al., 2010; Cleaver et al., 2011; Humphreys, Regan, River \& Thiara, 2005; Stover \& Kiselica, 2015). Ces situations complexes nécessitent également l'implication de plusieurs ressources d'aide aux expertises différentes, ce qui pose un défi de collaboration supplémentaire pour les intervenants (Mason \& O'Rinn, 2014). Cette étude qualitative, réalisée au Québec, explore les points de vue des bénéficiaires de services sur l'aide reçue et les pistes pour améliorer les interventions.

Le défi d'une aide intégrée et cohérente. Plusieurs recherches ont montré que les situations de cooccurrence posent des défis majeurs pour l'offre d'une aide intégrée et cohérente aux besoins des familles, car les expertises cliniques ont été développées dans des réseaux parallèles (Cleaver et al., 2011; Macy, Renz \& 
Pelino, 2013 ; Mason, DuMont \& Paterson, 2017a). Pour décrire ces défis de collaboration, certains auteurs parlent de «planètes distinctes» (Hester, 2011) ou de «schisme (clash) culturel» (Humphreys et al., 2005). Par exemple, les services en VC adoptent généralement une perspective féministe axée sur les rapports de pouvoir entre les hommes et les femmes (Lessard, Montminy, Lesieux, Flynn, Roy, Gauthier \& Fortin, 2015), alors qu'une perspective non genrée, mettant l'accent sur les facteurs individuels et la maladie, est plus souvent privilégiée en dépendance et en santé mentale (Humphreys et al., 2005). La présence de ces réseaux parallèles d'expertise complique l'offre de services intégrés et cohérents aux familles concernées. Pour les intervenants, il est alors plus simple de travailler chacun de leur côté dans leur champ d'expertise, car l'effort de concertation avec les autres secteurs d'expertise reste bien souvent peu soutenu par des mécanismes concrets au sein des différents organismes. Ces obstacles à la concertation entraînent, pour les familles vivant la cooccurrence, des enjeux de stigmatisation, d'accès aux services et de sécurité (Humphreys et al., 2005; Mason, et al., 2017b).

Même si certaines recherches ont documenté les taux de cooccurrence élevés ainsi que les défis de collaboration entre les différentes ressources concernées (Humphreys et al., 2005; Macy et al., 2013; Potito, Day, Carson \& O'Leary, 2009), la littérature scientifique demeure lacunaire concernant l'efficacité des programmes d'aide en situation de cooccurrence (Mason \& DuMont, 2015). Pourtant, une offre de services mieux intégrés permet de diminuer les symptômes posttraumatiques et la sévérité des PC chez les victimes (Corcozza et al., 2005). Une recension des écrits sur la cooccurrence de VC et de PC ou PSM montre également la nécessité d'améliorer la collaboration entre les ressources et l'intégration de l'aide offerte aux familles (Mason \& O'Rinn, 2014). Pour répondre à ce besoin, Mason et al. (2017a) ont élaboré un programme de formation intersectoriel en s'appuyant sur des entrevues auprès d'informateurs-clés et de victimes en situation de cooccurrence. L'évaluation du programme montre une amélioration significative des connaissances, attitudes et compétences des intervenants (Mason, Wolf, O’Rinn \& Ene, 2017b). Le travail conjoint avec les intervenants d'autres organismes et les discussions cliniques de cas ressortent comme les stratégies les plus efficaces pour améliorer la collaboration (Mason et al., 2017a).

Au Québec, la VC est définie comme une prise de contrôle sur un partenaire intime qui peut se manifester sous plusieurs formes et qui est généralement répétitive (gouvernement du Québec, 1995). Les services spécialisés en VC sont surtout offerts par des organismes communautaires, soit les maisons d'hébergement pour femmes victimes de $\mathrm{VC}$ et leurs enfants et les organismes qui viennent en aide aux conjoints exerçant la VC. Les établissements publics couvrent divers problèmes, incluant la VC, les PSM, les PC et la maltraitance des enfants. Ils offrent des services préventifs de première ligne et curatifs. Depuis 2007, la Loi sur la protection de la jeunesse reconnaît que la $\mathrm{VC}$ peut engendrer une maltraitance psychologique susceptible de nuire à la sécurité et au développement de l'enfant (gouvernement du Québec, 2018). L'exposition à la $\mathrm{VC}$ est l'une des formes de maltraitance les plus souvent prises en charge par les services de protection de l'enfance au Québec comme au Canada (Hélie, Collin-Vézina, Turcotte, Trocmé \& Girouard, 2017; Trocmé, Fallon, MacLaurin, Sinha, Black, Fast, ... Holroyd, 2010). La négligence et les PC des parents sont d'autres motifs très fréquents de prise en charge, d'ailleurs souvent cooccurrents à la VC (Lavergne, Hélie \& Malo, 2015; Mirick, 2014; Trocmé et al., 2010).

Cet état des connaissances souligne la nécessité de mieux connaître les actions susceptibles d'améliorer les services et de mieux répondre aux besoins complexes des familles en situation de cooccurrence, tout en 
reconnaissant que les personnes concernées mettent en place diverses stratégies de recherche d'aide. Selon Tanguy (2016), la recherche d'aide débute lorsqu'une personne décide de faire appel à des services professionnels, démarche favorisée ou non par le réseau informel. En situation de VC, la recherche d'aide peut être initiée pour différents besoins : soutien affectif, protection, démarches concrètes pour mettre un terme à la VC (Bilodeau, 1987). Mais la cooccurrence avec d'autres problèmes complique la recherche d'aide, car la diversité des besoins oblige souvent les personnes à contacter plusieurs ressources dont les services ne sont pas toujours bien coordonnés ou cohérents (Tanguy, 2016). Cette étude vise ainsi à documenter les stratégies de recherche d'aide (formelle et informelle) de parents et d'adolescents directement concernés par un vécu de cooccurrence de VC subie ou perpétrée et de PSM ou de PC dans les 2 dernières années ainsi que leurs recommandations pour améliorer les services d'aide utilisés. Bien qu'elles soient plus rares, quelques études qualitatives ont déjà questionné des femmes (Bohrman, Tennille, Levin, Rodgers \& Rhodes, 2017; Macy et al., 2013) ou des enfants (Templeton, Velleman, Hardy \& Boon, 2009) ayant un vécu de cooccurrence de $\mathrm{VC}$ et de PC. Par contre, la présente étude est, à notre connaissance, la première à s'intéresser à la fois au point de vue de pères, de mères et d'adolescents, à considérer divers types de cooccurrence et à documenter spécifiquement les stratégies de recherche d'aide.

\section{MÉTHODOLOGIE}

\section{Échantillonnage et caractéristiques des participants}

Le choix d'une approche qualitative et exploratoire, ancrée dans un paradigme interprétatif, s'avère approprié pour explorer le sens que les personnes donnent à leur expérience (Guba \& Lincoln, 1994), d'autant plus que l'état actuel des connaissances soulève davantage de questions que d'hypothèses eu égard à la cooccurrence de VC, de PSM et de PC. L'approche qualitative et exploratoire permet ainsi d'explorer la richesse et la diversité des points de vue des participants en lien avec le sujet de l'étude. Le recrutement des participants s'est fait grâce à la collaboration de plusieurs milieux de pratique, incluant les services publics généraux (Direction de la protection de la jeunesse (DPJ), santé mentale, dépendance) et les organismes communautaires pour les victimes et les auteurs de VC. Les intervenants des milieux de pratique ciblés présentaient le projet de recherche aux participants potentiels et, après avoir obtenu leur accord, transmettaient leurs coordonnées aux chercheures. Des étudiantes graduées et formées par les chercheures à la problématique et à la démarche de recherche étaient responsables de contacter les participants et de leur faire signer l'autorisation de participation à l'entrevue pour eux-mêmes ou leur adolescent lorsque ce dernier avait moins de 14 ans. Les adolescents de 12 et 13 ans signaient également un formulaire d'assentiment, conformément aux procédures en vigueur au Québec. Les étudiantes réalisaient les entrevues avec les participants. Chaque entrevue a été enregistrée, retranscrite et codifiée. Afin de s'assurer de l'uniformité de la codification, une procédure d'accord interjuges a été appliquée. L'analyse des données s'est faite par l'équipe de chercheures et d'étudiantes, avec le précieux soutien des partenaires de la pratique qui ont fourni des suggestions d'analyse et ont validé les résultats.

Pour participer à la recherche, les parents devaient avoir vécu eux-mêmes, dans les 2 dernières années, une situation de VC subie ou perpétrée accompagnée de PSM ou de PC. Les adolescents (12 à 17 ans) devaient avoir été exposés à cette cooccurrence dans les 2 dernières années. En cohérence avec l'approche 
exploratoire privilégiée, divers PSM ou PC ont été considérés; qu'ils soient diagnostiqués ou non, mais reconnus par les participants comme perturbant le fonctionnement familial. Parmi les plus souvent mentionnés par les participants, soulignons le syndrome de stress posttraumatique, l'anxiété, la dépression et la consommation de drogues ou d'alcool. L'échantillon inclut 43 participants (16 pères, 15 mères et 12 adolescents). La majorité d'entre eux (36) ne faisait pas partie de la même famille qu'un autre participant; sauf quelques mères et adolescents. Pendant le recrutement, le principe de diversification (Pirès, 1997) a été appliqué selon : le rôle dans la famille (père, mère, adolescent), le type de cooccurrence entre la VC et les problèmes associés, l'âge et le genre de l'adolescent (tableau 1). Les caractéristiques sociodémographiques des participants illustrent bien leurs conditions de vie difficiles. La majorité des mères interrogées et près de la moitié des pères vivent avec un revenu familial de moins de 30000 \$, ce qui est considéré être sous le seuil du faible revenu au Canada. Plusieurs sont monoparentaux et isolés socialement.

\section{Méthodes de collecte et d'analyse des données}

La principale méthode de collecte de données était l'entrevue semi-dirigée, soutenue par l'utilisation d'un outil sur la cartographie des services. Cet outil a permis d'identifier les sources d'aide formelle et informelle dont le répondant a pu bénéficier au cours des 2 dernières années ainsi que l'ordre de consultation des ressources. L'entrevue durait environ 90 minutes et abordait notamment les thèmes suivants : leur point de vue sur l'aide reçue, les aspects facilitants et les obstacles pour l'accès aux services ainsi que leurs recommandations pour améliorer l'offre d'aide. À la fin de l'entrevue, les participants complétaient un questionnaire sur leurs caractéristiques sociodémographiques. Une compensation de $30 \$$ a été remise à chacun. Le projet a été approuvé par les comités d'éthique des établissements concernés. La méthode d'analyse de contenu thématique (Braun \& Clarke, 2006) a été appliquée. La grille de codification a été validée par un comité de 7 experts en VC ( 3 chercheurs, 4 praticiens) impliqués dans les analyses. Afin de s'assurer que les résultats reflètent bien les réalités et les besoins des familles à l'égard de la cooccurrence, une synthèse des résultats a été retournée aux participants qui ont accepté d'être recontactés pour collaborer à leur validation (Lindlof \& Taylor, 2002).

\section{RÉSULTATS}

Les résultats montrent que, malgré les difficultés importantes auxquelles ils sont confrontés, les parents et les adolescents déploient plusieurs stratégies et font appel à une diversité de sources d'aide formelle et informelle. Les 2 premières sections présentent ces stratégies et les formes d'aide dont ils ont bénéficié. La dernière section fait état de leurs recommandations sur les améliorations possibles pour mieux répondre aux besoins des familles en situation de cooccurrence.

\section{Stratégies déployées}

Les adolescents décrivent d'une part les façons dont ils s'y prennent pour protéger leur mère, la fratrie ainsi qu'eux-mêmes. Par exemple, ils peuvent se sauver dans leur chambre ou ailleurs, s'interposer entre l'auteur de VC et la victime, appeler la police ou collaborer avec la DPJ. D'autre part, ils parlent de ce qu'ils font pour composer avec leur souffrance, notamment en extériorisant leurs émotions (cris, pleurs), en parlant 


\section{Tableau 1}

\section{Caractéristiques sociodémographiques des participants}

\begin{tabular}{|c|c|c|c|}
\hline Caractéristiques des participants & $\begin{array}{l}\text { Mères } \\
(n=15)\end{array}$ & $\begin{array}{l}\text { Pères } \\
(n=16)\end{array}$ & $\begin{array}{l}\text { Adolescents } \\
(n=12)\end{array}$ \\
\hline \multicolumn{4}{|l|}{ Type de cooccurrence } \\
\hline $\mathrm{VC} / \mathrm{PC}$ & & $18,8 \%(3)$ & $8 \%(1)$ \\
\hline $\mathrm{VC} / \mathrm{PSM}$ & $40 \%(6)$ & $6,3 \%(1)$ & $25 \%(3)$ \\
\hline $\mathrm{VC} / \mathrm{PSM} / \mathrm{PC}$ & $60 \%(9)$ & $75 \%(12)$ & $67 \%(8)$ \\
\hline \multicolumn{4}{|l|}{ Revenu } \\
\hline Moins de $30000 \$$ & $86,7 \%(13)$ & $43,8 \%(7)$ & $\mathrm{N} / \mathrm{A}$ \\
\hline 30000 à moins de $60000 \$$ & $6,7 \%(1)$ & $6,3 \%(1)$ & \\
\hline $60000 \$$ et plus & $6,7 \%(1)$ & $50 \%(8)$ & \\
\hline \multicolumn{4}{|l|}{ Scolarité } \\
\hline Études secondaires non complétées & $14,3 \%(2)^{*}$ & $12,5 \%(2)$ & $\mathrm{N} / \mathrm{A}$ \\
\hline Diplôme secondaire/professionnel & $40 \%(6)^{*}$ & $50 \%(8)$ & \\
\hline Études postsecondaires & $20 \%(3)^{*}$ & $37,5 \%(6)$ & \\
\hline \multicolumn{4}{|l|}{ Type de famille } \\
\hline Biparentale intacte & $6,7 \%(1)$ & $18,8 \%(3)$ & \\
\hline Biparentale recomposée & & $18,8 \%(3)$ & $8,3 \%(1)$ \\
\hline Monoparentale & $73,3 \%(11)$ & $12,5 \%(2)$ & $75 \%(9)$ \\
\hline Famille d'accueil (contacts réguliers parents/enfants) & $20 \%(3)$ & $50 \%(8)$ & $16,7 \%(2)$ \\
\hline \multicolumn{4}{|l|}{ Appartenance culturelle } \\
\hline \multicolumn{4}{|l|}{ Unique : } \\
\hline Québécoise/Canadienne & $84,6 \%(11)^{*}$ & $87,5 \%(14)$ & $58,3 \%(7)$ \\
\hline Autres** & & $12,5 \%(2)$ & \\
\hline Double appartenance ${ }^{* *}$ & $15,4 \%(2)^{*}$ & & $41,7 \%(5)$ \\
\hline \multicolumn{4}{|l|}{ Sexe des adolescents } \\
\hline Filles & $\mathrm{N} / \mathrm{A}$ & $\mathrm{N} / \mathrm{A}$ & $50 \%(6)$ \\
\hline Garçons & & & $50 \%(6)$ \\
\hline \multicolumn{4}{|l|}{ Âge des adolescents } \\
\hline $12-13$ ans & $\mathrm{N} / \mathrm{A}$ & $\mathrm{N} / \mathrm{A}$ & $33,3 \%(4)$ \\
\hline $14-15$ ans & & & $41,7 \%(5)$ \\
\hline $16-17$ ans & & & $25 \%(3)$ \\
\hline
\end{tabular}

* Une mère n'a pas répondu à la question sur la scolarité et 2 mères à la question sur l'appartenance culturelle. Pour ces variables, les \% sont calculés sur un total de 13 ou 14 mères.

** Latino-Américains (4), Caribéens-Antillais (2), Afrique subsaharienne (1), Premières Nations (1), Européen (1). N/A correspond aux variables non colligées auprès d'une catégorie de répondants. 
à quelqu'un de confiance, en se changeant les idées par des activités qu'ils aiment ou en se concentrant sur leur réussite scolaire.

«C'était tout le temps moi qui venais au milieu pour les séparer (...) j'appelais les voisins, j'ai déjà appelé la police, j'ai dû sortir par en arrière, par la fenêtre, des fois je cachais ma sœur dans la garde-robe parce que j'avais peur pour elle.» (Ado 7)

«J'essaie de rester positif. (...) de me changer les idées, je parle avec des amis, j'écoute de la musique, des choses comme ça.» (Ado 5)

De leur côté, les mères décrivent toutes être victimes de VC et mettent, elles aussi, en œuvre des stratégies qui visent leur protection et celle de leurs enfants : aller en maison d'hébergement, porter plainte à la police ou demander une ordonnance de protection, ne jamais laisser les enfants seuls avec le conjoint violent ou les confier à un proche de confiance le temps que le calme revienne à la maison ou que les problèmes associés à la VC soient moins aigus. Ce qu'elles font pour prendre soin d'elles (p. ex. jogging, yoga, aller au parc, écrire leur vécu) peut aussi les aider dans leur rôle de mère dans la mesure où cela contribue à leur mieux-être et à diminuer les effets de la VC sur leur santé mentale.

«Je [ne] l'ai jamais laissée toute seule avec [le conjoint violent], je la traînais partout. (...) des plans d'évacuation, acheter un deuxième cellulaire, cacher le deuxième cellulaire jusqu'à tant que ma décision de partir soit prise. (...) j'ai demandé de briser mon bail pour violence conjugale (...) quand je l'ai fait arrêter au mois de septembre il n'avait plus le droit de remettre les pieds dans le logement.» (Mère 1)

«Je [fais] du jogging, je prends soin de moi, je fais du yoga. (...) un moment donné, j'ai fait “non”. Moi, je vais sortir, j'ai une vie, je vais au parc (...)» (Mère 14)

Quant aux pères, bien que quelques-uns décrivent la dynamique de violence dans leur famille comme étant bidirectionnelle, la majorité s'identifie comme l'unique auteur de la VC. Les stratégies de ces pères consistent surtout à reconnaître le rôle qu'ils ont pu jouer dans les problèmes vécus et l'importance de poser des actions concrètes pour les résoudre. Leur souhait de devenir de meilleurs pères constitue souvent une source de motivation pour amorcer une demande d'aide et leur réseau informel peut d'ailleurs les inciter à consulter des services professionnels en lien avec les problèmes vécus. Ils soulignent également l'importance d'être assidus à leur programme d'intervention et d'appliquer les solutions qu'ils apprennent pour empêcher la $\mathrm{VC}$, parvenir à un mode de fonctionnement moins axé sur le contrôle de la conjointe et des enfants et s'affirmer plus sainement. Certains mentionnent aussi une stratégie de fuite dans le travail pour éviter les difficultés vécues à la maison. D'autres insistent sur la nécessité de changer leur réseau social pour arriver à diminuer leur PC.

«[En prison], j'ai décidé de changer ma vie (...) je me suis battu pendant un an pour [regagner] la garde de ma fille. (...) j'ai fait toutes les démarches inimaginables. (...) J'ai travaillé sur ma colère et (...) je n'ai jamais nié les actes que j'ai posés.» (Père 13)

\section{Formes d'aide reçue et priorisation des besoins}

Les différentes formes d'aide dont les participants ont bénéficié sont présentées au tableau 2. Les analyses indiquent que les adolescents mobilisent moins de ressources que les parents. Parmi les services obtenus au secteur privé, les pères sont les seuls à avoir mentionné les programmes d'aide aux employés. 
Ils sont aussi les seuls à bénéficier parfois du soutien de leurs collègues et supérieurs au travail. Le réseau informel figure parmi les premières sources d'aide consultées par les pères. Pour les mères, la porte d'entrée dans les services est davantage les services spécialisés en VC, en santé mentale ou en dépendance, ou encore le système de santé. Quant aux adolescents, la DPJ, la police ou la famille sont, pour la moitié d'entre eux, les premières ressources à intervenir auprès d'eux.

Les analyses croisées du discours des participants et des données recueillies par l'outil de cartographie montrent certaines nuances dans la manière dont les adolescents priorisent les besoins des membres de leur famille. Les adolescents qui ont reçu des services spécialisés en VC, souvent en maison d'hébergement, sont les seuls à parler de leurs propres besoins, par exemple en lien avec leur détresse ou leur santé mentale, alors que ceux qui n'ont pas reçu de services spécialisés en VC parlent uniquement des besoins perçus chez leurs parents. Ils nomment par exemple l'importance que leur père cesse sa consommation ou que leur mère reçoive des services en lien avec le stress vécu. Pour les jeunes ayant reçu des services en VC, la présence d'une intervenante jeunesse spécifiquement dédiée à leurs besoins a d'ailleurs été soulignée comme une force des services en maison d'hébergement. Certains, surtout ceux qui n'ont pas accès à une aide et une écoute dans leur réseau informel, insistent sur leur besoin d'être davantage impliqués ou informés des interventions offertes à leur famille. De même, lorsqu'ils n'ont pas reçu de services spécialisés en santé mentale, ils identifient comme prioritaire de s'occuper de leur propre santé mentale et de celle de leur mère. Des services plus proactifs sont d'ailleurs proposés pour aller au-devant des besoins des enfants qui dépendent bien souvent des démarches de leurs parents pour avoir accès à une aide.

«Ils nous ont dit : "Si vous avez besoin d'aide, vous me recontactez". Mais ils auraient dû mettre au moins 2-3 rencontres après qu'on soit parti, parce que tu sais toute femme et toute adolescente a besoin de parler après. Tu sais, moi je n'avais pas le courage de retourner là [maison d'hébergement].» (Ado 2)

Si les mères considèrent qu'il faut s'occuper en priorité des conséquences de la VC sur leur santé mentale, les pères insistent sur l'importance de l'accès à des services spécialisés tant en dépendance qu'en VC. Les pères expliquent que, même s'ils apprennent de nouveaux moyens pour agir sans violence, il est difficile de les mettre en pratique quand ils sont en état de consommation.

«Le stress [à travailler en premier] parce que ça te tue. Tu n'es même plus capable de réfléchir, c'est ça qui prime.» (Mère 11)

«La violence conjugale, c'est surtout en lien avec la consommation (...) Prioritaire... Si j'en vois un, je dirais que ce serait sur l'alcool.» (Père 1)

Toutefois, le travail prioritaire sur les PC est mentionné surtout par les pères qui ont consulté des services spécialisés en dépendance. De même, ceux qui ont consulté un organisme pour conjoints aux comportements violents sont les seuls à identifier en priorité le travail sur la VC (p. ex. gestion de la colère).

\section{Recommandations pour l'amélioration des services d'aide}

Les répondants des 3 catégories ont mentionné le besoin d'être soutenus et encouragés à partager leur vécu afin de se sentir compris. Il est primordial pour eux que l'intervenant les accueille sans jugement et s'intéresse à leur analyse des problèmes vécus. 


\section{Tableau 2}

Nombre de ressources d'aide formelle et informelle distinctes utilisées par les participants au cours des 2 dernières années

\begin{tabular}{|c|c|c|c|}
\hline Formes d'aide & Mères & Pères & Adolescents \\
\hline Aide formelle : nombre moyen de ressources/participant ${ }^{1}$ & 7,7 & 5,4 & 4,5 \\
\hline Maison d'hébergement & 10 & & 9 \\
\hline Organismes pour conjoints violents & 2 & 8 & \\
\hline Autre organisme en VC & & 3 & 8 \\
\hline DPJ & 12 & 11 & 9 \\
\hline Police & 13 & 7 & 6 \\
\hline Système de justice & 8 & 8 & 3 \\
\hline Prison & & 1 & \\
\hline CAVAC-IVAC & 13 & 1 & 1 \\
\hline Urgence-hôpital & 6 & 2 & 2 \\
\hline CISSS-CIUSSS première ligne & 13 & 5 & 5 \\
\hline Médecins de famille & 8 & 8 & \\
\hline Professionnel au privé et programme d'aide aux employés & 3 & 8 & 2 \\
\hline Psychiatrie ou pédopsychiatrie & 3 & 5 & 1 \\
\hline Ressource en dépendance & 6 & 7 & \\
\hline École & 2 & 1 & 4 \\
\hline Centre ou famille d'accueil & & & 3 \\
\hline Logement supervisé & 1 & & \\
\hline Autres organismes communautaires ${ }^{2}$ & 10 & 8 & 1 \\
\hline Centre d'appel & 6 & 3 & \\
\hline Aide informelle : nombre moyen de ressources/participant & 1,7 & 2,2 & 1,4 \\
\hline Famille & 9 & 16 & 7 \\
\hline Amis & 7 & 11 & 7 \\
\hline Entourage & 5 & 4 & 2 \\
\hline Collègues de travail et employeurs & & 3 & \\
\hline Internet & 4 & 4 & 1 \\
\hline Responsables religieux & 1 & 1 & \\
\hline Moyenne totale de ressources d'aide/participant & 9,3 & 7,6 & 5,9 \\
\hline
\end{tabular}

Note. 1. Certaines ressources d'aide ont été utilisées plus d'une fois par le même participant, surtout par les pères qui ont parfois interrompu leur suivi et l'ont repris plus tard, mais la ressource n'était alors comptabilisée qu'une seule fois.

2. Cette catégorie inclut les ressources pour la famille ou la santé mentale, les centres de femmes, les ressources pour hommes en difficulté et les centres de crise. 
«J'aurais aimé qu'on insiste sur le fait de parler. (...) je n'étais pas si forcée que ça d'en parler. (...) [dans le sens de] soutenue. D’être plus poussée, tu sais : "Vas-y! Vas-y en parler, ça va t'aider !" (..)

Pouvoir avoir quelqu'un qui vous écoute... (Intervieweur)

Puis qui me comprend.» (Mère 6)

«Ce n'est pas parce que je suis un enfant que... Ou un adolescent que je n'ai pas mon droit de parole ou de dire ce que moi j'ai vu, ce que je pense de la situation.» (Ado 8)

Les mères et les pères souhaiteraient aussi être davantage aidés dans leur rôle de parents avec des outils concrets, notamment pour développer leurs compétences à aborder la VC avec leur enfant.

«Une rencontre nous trois [mère, intervenante et enfant] (...) parce que (...) je ne suis pas outillée pour parler à mon enfant de choses qu'elle a vues ou entendues qu'elle n'aurait peut-être pas dû.» (Mère 13)

Alors que plusieurs mères mentionnent l'importance que les intervenants connaissent et considèrent les enjeux propres à la $\mathrm{VC}$, certains parents souhaitent aussi que les services tiennent davantage compte de leur expérience spécifique qui n'est pas toujours vécue ni interprétée de la même manière d'une situation à l'autre. Les participants considèrent d'ailleurs que l'intervention pourrait être mieux adaptée à leurs besoins spécifiques. Par exemple, une mère musulmane a mentionné son souhait que l'aide offerte puisse mieux rejoindre et travailler avec son conjoint en cohérence avec ses valeurs religieuses.

«Pour [les intervenantes] il y avait une seule possibilité, c'était de cesser la relation. Puis moi, c'était sûr que je trouvais ça dommage parce que je me dis qu'il devrait y avoir des services pour le couple. (...) C'est pour la protection de la femme, mais en même temps il y a un moyen de protéger la femme pour offrir de l'aide au conjoint (...) [qu'on] reconnai[sse] que l'homme a les capacités de changer.» (Mère 3)

L'accessibilité et la continuité de l'aide offerte par les différents services concernés sont également une priorité pour les participants à l'étude. En effet, la présence de problèmes cooccurrents semble compliquer l'accès des parents à certains services. Par exemple, les mères victimes de VC peuvent difficilement avoir accès à des services de santé mentale spécialisés si elles n'ont pas l'argent ou le répit parental nécessaire. Les mères expliquent que les PSM vécus peuvent limiter l'accès des femmes à d'autres services, leur situation étant perçue comme trop complexe. Pour les participants, la continuité de l'aide inclut aussi l'aide informelle et les ressources d'aide d'autres domaines que les PSM ou PC. En ce sens, certaines femmes mentionnent un besoin d'entraide entre femmes qui ont un vécu semblable, ce qui leur permettrait de recréer leur réseau social. De même, quelques adolescents, surtout parmi les plus âgés, souhaiteraient plus de soutien dans les aspects concrets de leur vie qui les aideront à s'émanciper du contexte familial difficile (p. ex. recherche d'emploi, développement d'un réseau social). Quant aux pères, l'accès rapide à des services d'urgence s'avère essentiel pour désamorcer les crises et éviter les risques de violences létales envers eux-mêmes ou leurs proches. Des pères mentionnent que le fait «d'être dans le système de santé et de services sociaux»et d'avoir un médecin de famille peut favoriser l'accès plus rapide à des services spécialisés.

«J'étais vidée, j'avais tout perdu, je n'avais plus de qualité de vie [j'aurais aimé avoir] de l'aide de gardiennage (...) une personne aussi qui m'aide à me ressourcer.» (Mère 7)

«Du soutien émotionnel, pas tout le temps les menottes (...) Il pourrait y avoir des services pour des situations d'aide, d'urgence.» (Père 12) 
De plus, puisque chaque ressource se spécialise dans un domaine en particulier, les parents et les adolescents expliquent que les liens entre les problèmes vécus et leur impact sur l'exercice de la maternité ou de la paternité ne sont pas toujours faits par les intervenants. Certains déplorent aussi le fait de devoir composer avec des décisions incohérentes des différents tribunaux impliqués dans leur situation, comme une interdiction de contact en droit criminel en raison de la VC et une garde partagée en droit de la famille. Ils revendiquent une meilleure communication entre les tribunaux et une prise en compte de la VC dans les décisions liées à la garde des enfants. Certaines décisions peuvent prioriser les problèmes associés (PSM ou PC), en omettant de considérer la VC qui perdure parfois au-delà de la séparation des parents.

«Les trois [tribunal de la jeunesse, Cour civile et Cour criminelle] ne se parlent pas. (...) [1'avocat de l'exconjoint a fait enlever une preuve du dossier criminel] c'est tellement triste parce qu'au bout du compte, ce sont nous les victimes qui vont paraître pour les personnes qui sont menteuses. Et c'est extrêmement dur. (...) avec le fardeau d'assurer toute seule la sécurité de mes enfants (...) Et puis, monsieur se sert de la garde des enfants (...) pour faire du harcèlement à travers les tribunaux.» (Mère 15)

D'ailleurs, les 3 catégories de répondants ont souligné l'importance de sensibiliser davantage la population générale et les professionnels des services sociaux et du système judiciaire à la VC et aux problèmes associés. Pour eux, la continuité et la cohérence de l'aide ne peuvent être atteintes sans une meilleure connaissance des problèmes qu'ils vivent et des ressources d'aide. Ils croient aussi qu'il faut plus de prévention, pour éviter que la situation familiale dégénère entraînant des problèmes cooccurrents.

«C'est tabou [la VC] il faudrait que ça le soit beaucoup, beaucoup moins.» (Mère 13)

«Il y a des ressources... Mais on n'est pas informés. (...) de ne pas hésiter à aller chercher de l'aide. (...) mon rôle, comme parent [sera] d'éduquer mes enfants à ça.» (Père 1)

\section{DISCUSSION}

Les résultats de cette étude montrent les défis importants de l'offre d'aide aux familles en situation de cooccurrence. Puisque la conscientisation de ses besoins est un processus continu pouvant être favorisé par l'accès aux services spécialisés en $\mathrm{VC}$, en dépendance ou en santé mentale, il s'avère crucial que les différentes ressources se connaissent bien entre elles et soient à l'aise d'accueillir et même favoriser le dévoilement de la VC et des problèmes associés. Cet accueil devrait se faire sans jugement et avec sensibilité au vécu des familles (Mason et al., 2017a). Une meilleure évaluation des problèmes cooccurrents et des besoins en cause pourrait favoriser un accès plus rapide à des services spécialisés, permettant ainsi d'éviter que la situation familiale se détériore encore davantage au point que les parents en viennent à perdre la garde de leurs enfants, comme c'est le cas de $20 \%$ des mères et de $50 \%$ des pères rencontrés dans cette recherche.

Si les recommandations faites par les participants à la recherche semblent toutes importantes, nos résultats convergent avec la recommandation principale de Mason et O'Rinn (2014) quant à la nécessité d'améliorer la continuité et la cohérence de l'aide offerte aux familles. Lorsque les différents intervenants et organismes portent des visions trop différentes des problèmes et des solutions, il devient plus difficile d'offrir une aide intégrée et cohérente aux familles en situation de cooccurrence. Il importe alors de replacer au cœur des débats la vision et les besoins des personnes directement concernées, d'où l'importance de la présente étude. D'autres recherches (p. ex. Mason \& DuMont, 2015) ont montré que les victimes de VC 
déplorent l'approche biomédicale qui se limite à poser un diagnostic ou prescrire un traitement, car une telle approche omet de considérer l'enchevêtrement complexe entre l'expérience de la VC et des problèmes cooccurrents. En effet, le stress de vivre dans un environnement violent et imprévisible peut accentuer les risques de développer des PC ou PSM, et ces derniers augmentent également la vulnérabilité à subir de la VC (Mason \& DuMont, 2015).

Des consultations récentes, réalisées auprès de 57 experts des différentes ressources d'aide concernées au Québec montrent que la concertation clinique reste un défi de taille (Dumont, 2018), et ce, en dépit de la réforme majeure du réseau public québécois (Loi 10 adoptée en 2015) qui visait justement une plus grande accessibilité et une meilleure continuité de services. Comme la VC est une problématique transversale, il faudrait que les nouveaux établissements régionaux, nommés Centres intégrés (parfois universitaires) de santé et de services sociaux (CISSS ou CIUSSS), désignent des intervenants pivots en VC dans leurs différents secteurs de pratique (DPJ, première ligne en enfance-famille-jeunesse, en santé mentale et en dépendance), afin d'assurer une concertation efficace entre ces secteurs ainsi qu'avec les ressources communautaires. De plus, les formations offertes aux intervenants sur la VC, les PSM et les PC n'abordent généralement pas les liens entre ces problèmes et sont très rarement offertes en intersectorialité comme recommandé par Mason et al. (2017a). C'est pourquoi la formation continue et intersectorielle des intervenants concernés ainsi que les modèles de concertation clinique novateurs s'avèrent être des stratégies intéressantes à mobiliser, qui pourraient aussi faire l'objet de recherches évaluatives (Mason et al. 2017 a, 2017b; Lessard, Drouin, Germain, Alvarez-Lizotte \& Turcotte, 2014).

Les stratégies déployées par les personnes en situation de cooccurrence fournissent des pistes d'action intéressantes à mobiliser dans l'intervention. Les résultats de notre étude montrent que ces stratégies et la porte d'entrée dans les services formels diffèrent pour les mères, les pères et les adolescents en situation de cooccurrence, et ce, tant pour le nombre de ressources mobilisées que le type de services. Ces résultats soulèvent plusieurs questions qu'il serait pertinent d'approfondir dans les recherches futures. Par exemple, si les mères font moins appel à leur réseau informel (famille et amis) que les pères, est-ce parce que la VC a conduit ces victimes à s'éloigner de leurs proches? En effet, parmi les manifestations de contrôle coercitif qui caractérisent plusieurs situations de VC, on retrouve notamment les stratégies du conjoint violent qui visent à dénigrer la victime dans son rôle parental et à l'isoler de son réseau d'aide informel (Stark, 2014). De plus, les préjugés sociaux sur les capacités parentales des mères victimes de VC qui présentent des PSM ou PC entraînent chez elles une crainte d'être jugées comme de mauvaises mères par les intervenants professionnels ou par leur famille et amis (Bohrman et al., 2017). D'ailleurs, leur crainte que la DPJ leur retire la garde des enfants si elles dévoilent la violence subie et les problèmes cooccurrents serait tout à fait légitime, puisqu'une étude récente montre que la présence de cooccurrence augmente le risque de placement des enfants (Lavergne et al., 2018). Les intervenants auprès des femmes/mères en situation de cooccurrence devraient privilégier une approche globale qui tient compte et traite les traumas, ce qui constitue une condition essentielle pour les soutenir efficacement dans leur rôle de mères (Huntington, Moses \& Veysey, 2005 ; Mason et al., 2017 a; Waldman-Levi, Finzi-Dottan \& Weintraub, 2015).

Quant aux pères, nos résultats montrent que très peu d'interventions ciblent spécifiquement la paternité tout en tenant compte de la violence exercée ou des problèmes associés. Pourtant, une étude de Stover (2015) montre qu'un programme sur la paternité privilégiant une approche globale des problèmes vécus est plus 
efficace qu'un traitement individuel de la toxicomanie pour maintenir les hommes engagés dans leur démarche et pour diminuer la $\mathrm{VC}$ et la maltraitance des enfants. Tout comme les mères, ils peuvent être soumis à des préjugés sociaux susceptibles de les décourager à faire une demande d'aide. Par ailleurs, puisque les hommes rencontrés dans la présente recherche consultent davantage leurs collègues de travail et les programmes d'aide aux employés, on peut se demander si ce secteur est suffisamment mis à contribution dans les efforts de prévention de la VC. Toutefois, les pères participants étaient tous en contact avec des ressources d'aide, ce qui s'explique par la stratégie de recrutement privilégiée et qui ne reflète pas nécessairement la réalité de l'ensemble des hommes/pères en situation de cooccurrence. En effet, il importe de rappeler les obstacles structurels auxquels autant les mères que les pères se butent, en particulier les conditions socioéconomiques précaires et l'isolement social, qui constituent des barrières supplémentaires pour l'accès aux services; barrières auxquelles Bohrman et al. (2017) recommandent de s'attaquer.

Concernant les adolescents, puisque notre étude montre qu'ils reçoivent moins d'aide que leurs parents, que les premiers services à intervenir dans leur vie sont souvent des services d'urgence lorsque leur sécurité est déjà compromise (police, DPJ) et qu'ils demandent des services plus proactifs, il semble prioritaire de modifier l'offre d'aide pour s'assurer de les impliquer davantage. Est-ce que les premiers intervenants avec lesquels ils sont en contact vérifient systématiquement s'ils ont, dans leur réseau informel, un adulte de confiance à qui ils peuvent parler de leur vécu? Dans le cas où les enfants sont plus isolés, il serait important de leur offrir de l'aide, en expliquant bien aux parents l'importance de services spécifiquement adaptés aux besoins des enfants. Cela renforce également l'importance de la prévention et de la sensibilisation revendiquée par les participants à cette recherche, en particulier dans le milieu scolaire. Par ailleurs, si certaines stratégies utilisées par les adolescents peuvent les maintenir dans un rôle d'enfants "parentifiés" (p. ex. protéger le parent victime ou la fratrie), elles rappellent l'importance de considérer les enfants et les adolescents comme des acteurs non passifs, toujours à la recherche de solutions pour favoriser leur mieuxêtre et celui des membres de leur famille (DeBoard-Lucas \& Grych, 2011; Gorin, 2004; Templeton et al., 2009). D'ailleurs, certaines stratégies des adolescents, comme le fait de se confier à un adulte de confiance ou de s'occuper positivement, devraient être soutenues et encouragées.

Cette recherche a permis de mieux comprendre les stratégies de recherche d'aide des familles en situation de cooccurrence de la VC et d'un PSM ou PC ainsi que leurs recommandations pour l'amélioration des services, ce que peu d'études ont fait jusqu'ici. Puisque la méthode de recrutement a permis de rencontrer uniquement des personnes qui ont consulté au moins un organisme d'aide, que les trajectoires de services sont différentes d'un participant à l'autre et que l'organisation des services comporte des spécificités dans chaque pays ou région, les résultats ne peuvent être transposés à l'ensemble des familles en situation de cooccurrence. De plus, les expériences de ces personnes et les liens qu'elles perçoivent entre les problèmes cooccurrents varient beaucoup d'une famille à l'autre (Alvarez-Lizotte, Lessard, Lévesque, Lavergne \& Dumont, 2018), ce qui peut influencer le discours des participants sur leurs besoins en matière d'aide. L'échantillonnage s'est appuyé sur le principe de diversification qui est particulièrement important en recherche qualitative (Pirès, 1997) et constitue donc une force de l'étude. Toutefois, certaines dimensions de l'analyse n'ont pu atteindre une saturation empirique. Des recherches futures s'intéressant à une catégorie de répondants à la fois (pères, mères ou adolescents) permettraient d'avoir un échantillon plus grand d'une même catégorie de répondants et, ainsi, approfondir l'analyse des points de vue et recommandations des familles sur les services 
qui leur sont offerts. D'où l'importance de poursuivre le développement des connaissances sur les vécus complexes des familles confrontées à la cooccurrence de VC et d'un PSM ou PC et sur les interventions les plus efficaces pour leur venir en aide.

\section{RÉFÉRENCES}

Alvarez-Lizotte, P., Lessard, G., Lévesque, S., Lavergne, C. \& Dumont, A. (2018). Expériences et besoins de mères, de pères et d'adolescents vivant à la fois de la violence conjugale et des difficultés liées à la santé mentale ou à la consommation des parents. CRI-VIFF : Fiche synthèse Recherche, 21, 1-4.

Bohrman, C., Tennille, J., Levin, K., Rodgers, M. \& Rhodes, K. (2017). Being superwoman: low income mothers surviving problem drinking and intimate partner violence. Journal of family violence, 32(7), 699-709. doi:10.1007/s10896-017-9932-5

Braun V. \& Clarke V. (2006). Using thematic analysis in psychology. Qualitative Research in Psychology 3: 77-101. doi:10.1191/1478088706qp063oa

Bromfield, L., Lamont, A., Parker, R. \& Horsfall, B. (2010). Issues for the safety and wellbeing of children in families with multiple and complex problems. The co-occurrence of domestic violence, parental substance misuse, and mental health problems. Melbourne, Vic. : Australian Institute of Family Studies.

Choenni, V., Hammink, A. \& van de Mheen, D. (2017). Association between substance use and the perpetration of family violence in industrialized countries: a systematic review. Trauma, Violence, \& Abuse, 18(1), 37-50. doi:10.1177/1524838015589253

Cleaver, H., Unell, I. \& Aldgate, J. (2011). Children's Needs-Parenting Capacity. Child abuse: Parental mental illness, learning disability, substance misuse and domestic violence. London: TSO.

Cocozza J.J., Jackson, E.W., Hennigan, K., Morrissey, J.P., Reed, B.G., Fallot, R. et al. (2005). Outcomes for Women with CoOccurring Disorders and Trauma: Program-Level Effects. Journal of Substances Abuse Treatment, 58: $43-54$.

DeBoard-Lucas, R. L. \& Grych, J. H. (2011). Children's perceptions of intimate partner violence: Causes, consequences, and coping. Journal of Family Violence, 26(5), 343. DOI 10.1007/s10896-011-9368-2

Dumont, A. (2018). Violence conjugale et problèmes associés : recommandations d'expertes de divers milieux de pratique s'appuyant sur des résultats de recherche. Fiche synergie recherche-pratique \# 7. CRI-VIFF. Repéré à https://www.criviff.qc.ca/

Estefan, L. F., Coulter, M. L., VandeWeerd, C. L., Armstrong, M. \& Gorski, P. (2013). Relationships Between Stressors and Parenting Attitudes in a Child Welfare Parenting Program. Journal of Child and Family Studies, 22, 199-208.

Galvani, S. (2004). "Responsible disinhibition: Alcohol, men and violence to women", Addiction Research \& Theory, vol. 12, n 4, p. 357-371.

Gorin, S. (2004). Understanding What Children Say: Children's experiences of domestic violence, parental substance misuse and parental health problems. London: National Children's Bureau.

Gouvernement du Québec. (1995). Politique d'intervention en matière de violence conjugale. Prévenir, dépister, contrer la violence conjugale. Québec : gouvernement du Québec, Ministère de la santé et des services sociaux.

Gouvernement du Québec. (2018). Loi sur la protection de la jeunesse. Repéré à http://www2.publicationsduquebec.gouv.qc.ca/ dynamicSearch/telecharge.php?type=2\&file=/P $34 \quad$ 1/P34 1.html

Guba, E.G. \& Lincoln, Y.S. (1994). «Competing paradigms in qualitative research». In Denzin, N. \& Lincoln, Y.S. (eds), Handbook of Qualitative Research, Thousand Oaks, CA: Sage

Hélie, S., Collin-Vézina, D., Turcotte, D., Trocmé, N. \& Girouard, N. (2017) Étude d’incidence québécoise sur les situations évaluées en protection de la jeunesse en 2014 (ÉIQ-2014) : Rapport final. Remis au ministère de la Santé et des Services sociaux. Repéré à http://cwrp.ca/sites/default/files/publications/en/eiq-2014_rapport_final.pdf

Hester, M. (2011). The Three Planet Model: Towards an Understanding of Contradictions in Approaches to Women and Children's Safety in Contexts of Domestic Violence. British Journal of Social Work, 41, 837-853. doi:10.1093/bjsw/bcr095

Humphreys, C., Regan, L., River, D. \& Thiara, R. (2005). Domestic Violence and Substance Use: Tackling Complexity. British Journal of Social Work, 35, 1303-1320. doi:10.1093/bjsw/bch212

Huntington, N., Moses, D. J. \& Veysey, B. M. (2005). Developing and implementing a comprehensive approach to serving women with co-occurring disorders and histories of trauma. Journal of community psychology, 33(4), 395-410. doi:10.1002/jcop.20059

Lavergne, C. Hélie, S. \& Malo, C. (2015). Exposition à la violence conjugale : profil des enfants signalés et réponse aux besoins d'aide des familles. Revue de psychoéducation, 2, 245-267. doi:10.7202/1039255ar

Lessard, G., Drouin, M.-E., Germain, A.-S., Alvarez-Lizotte, P. \& Turcotte, P. (2014). Concerted pratice-based actions in intimate partner and family violence: when the children's well-being is the central concern. Social sciences, 3, 650-671. doi:10.3390/ socsci3040650

Lessard, G., Montminy, L., Lesieux, E., Flynn, C., Roy, V., Gauthier, S. \& Fortin, A. (2015). Les violences conjugales, familiales et structurelles : vers une perspective intégrative des savoirs. Enfances, Familles, Générations, 22, 1-26. doi :10.7202/1031116ar 
Lindlof, T. R. \& Taylor, B.C. (2002). Qualitative communication research methods (2nd ed.). Thousand Oaks, CA: Sage.

Macy, R. J., Renz, C. \& Pelino, E. (2013). Partner Violence and Substance Abuse Are Intertwined: Women's Perceptions of ViolenceSubstance Connections. Violence Against Women, 19(7), 881-902. doi:10.1177/1077801213498208

Mason, R. \& DuMont, J. (2015). Advancing our knowledge of the complexity and management of intimate partner violence and co-occurring mental health and substance use problems in women. F1000 Prime Reports, 7. doi:10.12703/P7-65

Mason, R. \& O’Rinn, S.E. (2014). Co-occurring intimate partner violence, mental health, and substance use problems: a scoping review. Global Health Action, 7, 1-17. doi:10.3402/gha.v7.24815

Mason, R., DuMont, J. \& Paterson, M. (2017a). Enhancing Collaboration Between Children's Aid Societies and Adult Mental Health Services. Prepared for the Ministry of Children and Youth Services. Government of Ontario, Canada.

Mason, R. Wolf, M., O'Rinn, S. \& Ene, G. (2017b). Making connections across silos : intimate partner violence, mental health, and substance use. BMC Women's Health, 17 : 29. doi:10.1186/s12905-017-0372-4

Mirick, R. G. (2014). Engagement in Child Protective Services: The Role of Substance Abuse, Intimate Partner Violence and Race. Child and Adolescent Social Work Journal, 31, 267-279. doi: 10.1007/s10560-013-0320-6

OMS (2006). «Dossier de l'OMS sur l'alcool et la violence à l'égard du partenaire intime», Agence de santé publique du Canada - Promotion de la $<$ https://www.canada.ca/fr/sante-publique/services/promotion-sante/arretons-violence-familiale/ressourcesprevention/femmes/dossier-alcool-violence-egard-partenaire-intime.html>, consulté le ler mai 2018.

Pirès, A. (1997). «Échantillonnage et recherche qualitative : essai théorique et méthodologique». Dans Poupart et al., La recherche qualitative. Enjeux épistémologiques et méthodologiques (pp. 113-169). Montréal, Canada : Gaëtan Morin.

Potito, C., Day, A., Carson, E. \& O’Leary, P. (2009). Domestic Violence and Child Protection: Partnerships and Collaboration. Australian Social Work, 62(3), 369-387. doi :10.1080/03124070902964657

Stark, E. (2014). Un re-présentation des femmes battues. Contrôle coercitif et défense de la liberté. Dans M. Rinfret-Raynor, É. Lesieux, M.-M. Cousineau, S. Gauthier et É. Harper (eds.), Violences envers les femmes : réalités complexes et nouveaux enjeux dans un monde en transformation (pp. 33-51). Québec, Canada : Les presses de l'Université du Québec.

Stover, C. S. (2015). Fathers for change for substance use and intimate partner violence: Initial community pilot. Family process, 54(4), 600-609. doi: 10.1111/famp.12136

Stover, C. S. \& Kiselica, A. (2015). Hostility and substance use in relation to intimate partner violence and parenting among fathers. Aggressive Behavior, 41(3), 205-213. doi:10.1002/ab.21548

Tanguy, A. (2016). La recherche d'aide des femmes victimes de violence conjugale. Fiche synthèse Recension, TRAJETVI. Repéré à http://trajetvi.ca/publication/la-recherche-d-aide-des-femmes-victimes-de-violence-conjugale

Templeton, L., Velleman, R., Hardy, E. \& Boon, S. (2009). Young people living with parental alcohol misuse and parental violence: "Noone has ever asked me how I feel in any of this". Journal of Substance Use, 14(3-4), 139-150. doi:10.1080/14659890802624287

Trocmé, N., Fallon, B., MacLaurin, B., Sinha, V., Black, T., Fast, ... Holroyd, J. (2010). Canadian Incidence Study of Reported Child Abuse and Neglect-2008. Ottawa: Public Health Agency of Canada.

Waldman-Levi, A., Finzi-Dottan, R. \& Weintraub, N. (2015). Attachment security and parental perception of competency among abused women in the shadow of PTSD and childhood exposure to domestic violence. Journal of Child and Family Studies, 24(1), 57-65. doi : 10.1007/s10826-013-9813-3 